Ann. Biol. anim. Bioch. Biophys., I970, 10 (2), I95-202.

\title{
QUELQUES OBSERVATIONS SUR LA REPRODUCTION DU LIÈVRE EUROPÉEN (LEPUS EUROPAEUS PALLAS) EN CAPTIVITÉ
}

\author{
Lise MARTINET, J.-J. LEGOUIS et B. MORET \\ Station centrale de Physiologie animale, \\ Centre national de Recherches zootechniques, 78 -Jouy-en-Josus \\ Institut national de la Recherche agronomique
}

\section{RÉSUMLÉ}

A partir d'observations réalisées pendant 3 années d'élevage, quelques renseignements concernant la reproduction du Lièvre en captivité ont pu être rassemblés. La durée de la saison sexuelle s'étend de janvier à août-septembre, comme dans la nature. La taille de la portée passe par un maximum de mai à juillet.

La durée de la gestation est de 40 à 4 I jours. Une superfoetation naturelle, résultant d'accouplements féconds pendant la gestation, s'observe dans 60 p. Ioo des mise bas; cette fréquence serait beaucoup plus forte que dans la nature.

\section{INTRODUCTION}

Si depuis quelques années plusieurs travaux ont décrit les cycles de reproduction du Lièvre européen dans la nature (en Russie, KoLosov, I94I ; en Allemagne, Stieve, I952 ; en Pologne, Raczynski, ig64; en Nouvelle-Zélande, Flux, i967), les données en élevage sont, par contre, très rares à cause des difficultés rencontrées pour maintenir ces animaux en captivité ; seul HEDIGER et ses collaborateurs (I954 à I967) ont publié quelques résultats sur la biologie sexuelle du Lièvre en captivité.

Le Lièvre européen montre un cycle saisonnier de reproduction de janvier à septembre; la fertilité des individus estimée par le poids des testicules et la taille de la portée passe par un maximum d'avril à juin.

La durée de la gestation a été depuis toujours très discutée ; en effet, l'existence d'une superfotation, c'est-à-dire d'un accouplement fertile pendant la gestation, connue depuis l'antiquité a gêné les observations; il existe souvent une confusion entre la durée de la gestation et l'intervalle de temps séparant deux mise bas. Il est 
cependant admis maintenant que la gestation chez cette espèce est de 42 jours environ (BLOCH et al., 1954).

L'existence de superfotation semble rare dans la nature; FLUX (I967) ne 1'observe que chez 3 hases sur 428 gestantes; RACYNSKI (I964), REYNOLDS (I950) n'en observent aucune.

Par contre, en captivité, des accouplements fertiles précédant la mise bas de quelques jours semblent fréquents (Br,OCH et al., I954).

\section{MATÉRIEL, ET TECHNIQUES}

Les observations réunies dans ce travail proviennent de 3 années d'élevage et sont faites à partir de Lièvres achetés en Tchécoslovaquie et de leurs descendants.

L'étude porte sur I 2 à I6 couples en moyenne par année avec un total pour les 3 années de 360 levrauts nés de I 54 portées.

Les animaux sọt conservés en parquet à l'extérieur; ils sont — soit par couple pendant toute la durée de la saison de reproduction, - soit isolés, le mâle n'étant introduit dans la cage de la femelle que pour une durée de 24 heures.

Chaque jour les signes de poursuites, précédant les accouplements, sont enregistrés ; les jeunes sont pesés à la naissance et à un mois au moment du scvrage.

\section{RÉSULLTATS}

\section{Durée de la saison sexuelle}

Comme dans la nature, la reproduction a lieu de janvier à septembre environ.

Le début de la saison sexuelle semble assez fixe; en effet en ig67 et I968, les testicules de tous les mâles sont descendus en position scrotale pendant la première semaine de décembre.

Les premières naissances ont eu lieu :

- le 22 février I967;

- le 24 janvier I968;

- le 3 février ig69.

En I967, les couples n'ont été formés qu'au début de janvier, ce qui explique la date tardive de la première mise bas ; alors qu'en Ig68 et Ig69 ils l'étaient dès le I5 novembre.

Les dernières naissances ont eu lieu:

- en I967, le I4 septembre;

- en I968, le 26 aôit ;

- en I969, le I7 octobre seulement, grâce à deux mises bas tardives suivant un arrêt presque total de la reproduction, du I 5 juillet à la fin septembre.

\section{Age à la maturité sexuelle}

Si les jeunes nés de janvier à avril ne semblent pas atteindre la maturité sexuelle avant l'hiver suivant, c'est-à-dire entre 9 et I 2 mois, par contre, les jeunes nés à partir de juin se reproduisent dès 1'âge de 6 mois. 
IA REPRODUCTION DU LIÈVRE EN CAPTIVITÉ

I97

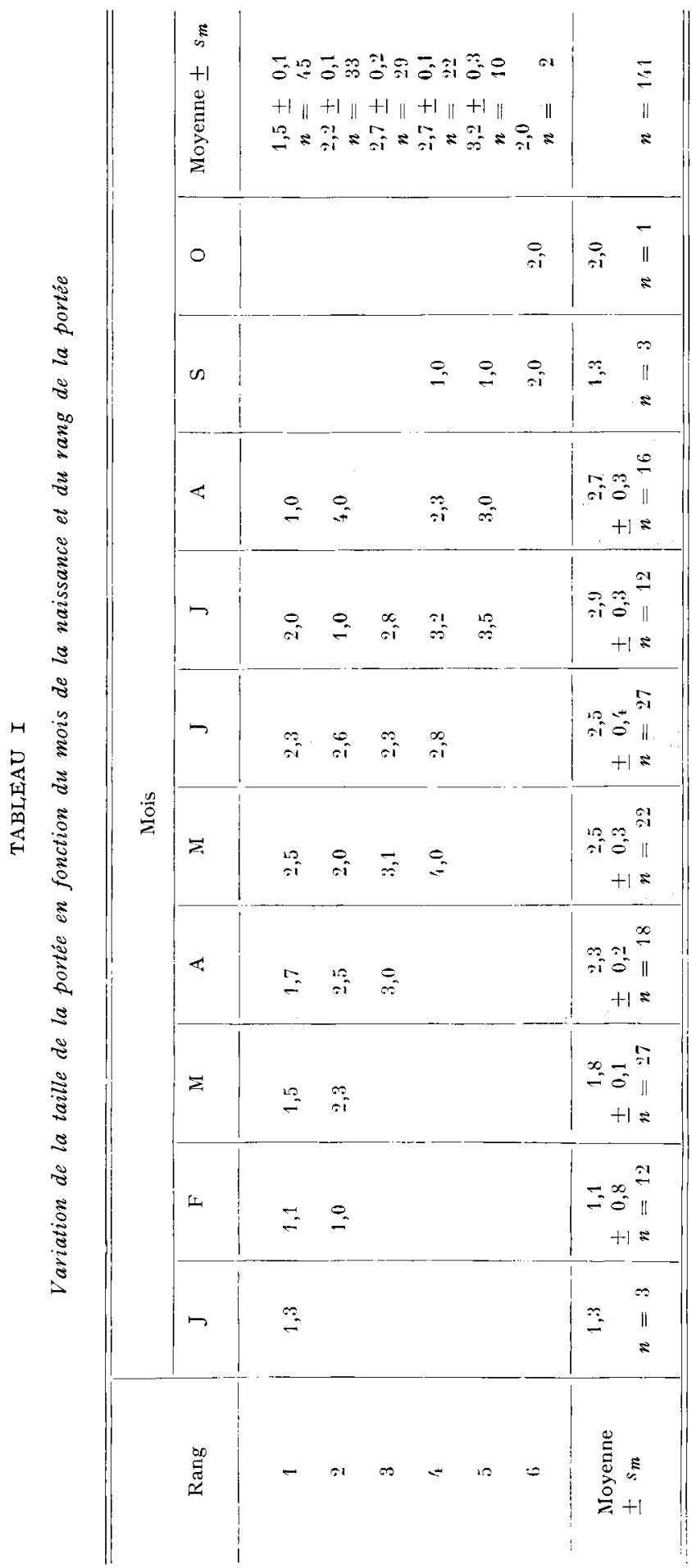




\section{Production de jeunes}

Le nombre maximum de portées par femelle et par an a été de 6 , la plus grande fréquence étant de 3 ou 4 .

Le nombre maximum de jeunes nés par femelle et par an a été de I7 en 5 portées ; le plus souvent il se situe entre 8 à $\mathrm{I} 2$ levrauts.

\section{La taille de la portée}

La plus forte portée obtenue a été de 5 ; mais des portées de 6 ou 7 ont été observées dans d'autres élevages (BIADr, communication personnelle).

Comme le montre le tableau $\mathrm{I}$, elle dépend du rang de la portée au cours de l'année, mais aussi du mois de la naissance. Les moyennes les plus élevées se situent d'avril à juillet pour les troisième, quatrième et cinquième portées. L'analyse statistique montre que les différences observées sont significatives $(\mathrm{P}<0, \mathrm{or})$.

Il ne semble pas que la taille de la portée soit plus faible pour les femelles en première année de reproduction ; mais le nombre de comparaisons possibles n'est pas assez élevé pour pouvoir l'affirmer.

\section{Croissance des jeunes au cours du $1^{\mathrm{er}}$ mois}

Le poids des levrauts à la naissance (tabl. 2) est excessivement variable (amplitude $=40$ à I $80 \mathrm{~g}$ ) ; la moyenne pour 283 jeunes pesés est de I I $3,2 \pm$ I5,6 g.

TABLEAU 2

Poids des levrauts à la naissance en fonction de la taille de la portée et de la date de la mise bas

\begin{tabular}{|c|c|c|c|c|}
\hline $\begin{array}{l}\text { Taille de } \\
\text { la portée }\end{array}$ & \multicolumn{2}{|c|}{$\begin{array}{c}\text { Animaux nés en janvier, février } \\
\text { mars }\left(\mathrm{g} \pm s_{m}\right)\end{array}$} & \multicolumn{2}{|c|}{$\begin{array}{c}\text { Animaux nés d'avril à octobre } \\
\left(g \pm s_{m}\right)\end{array}$} \\
\hline 1 & $114,3+7,7$ & $n=23$ & $124,3 \pm 7,0$ & $n=20$ \\
\hline 2 & $106,4 \pm 9,2$ & $n=18$ & $116,4 \pm 5,3$ & $n=48$ \\
\hline 3 & $60,4 \pm \quad 1,4$ & $n=9$ & $110,9 \pm 2,8$ & $n=90$ \\
\hline 4 & $88,3 \pm 5,7$ & $n=12$ & $107,4 \pm 4,6$ & $n=48$ \\
\hline 5 & $=$ & $n=0$ & $108,9 \pm 6$ & $n=15$ \\
\hline
\end{tabular}

Ce poids diminue de manière significative $(\mathrm{P}<0,05)$ quand la taille de la portée augmente ; d'autre part, il semble que les levrauts nés à partir d'avril soient plus gros que ceux nés pendant les trois premiers mois d'hiver; la différence est significative pour les portées de 3 ou 4 jeunes.

Le poids des levrauts, à un mois, au moment du sevrage, se situe autour d'un $\mathrm{kg}$; il diminue de manière hautement significative en fonction de la taille de la portée à la mise bas $(\mathrm{P}<0, \mathrm{OI})$ (tabl. 3). 
TABLEAAU 3

Poids des levrauts au sevrage

en fonction de la taille de la portée à la mise bas

\begin{tabular}{|c|c|c|}
\hline $\begin{array}{l}\text { Taille } \\
\text { de la portée }\end{array}$ & Nombre d'animaux & $\begin{array}{l}\text { Poids all sevrage } \\
\quad\left(\mathrm{g} \pm s_{m}\right)\end{array}$ \\
\hline 1 & 19 & $1022 \pm 53$ \\
\hline 2 & 27 & $970 \pm 38$ \\
\hline 3 & 31 & $908 \pm 25$ \\
\hline 4 & $\because 0$ & $80 x \pm 5 t$ \\
\hline 5 & 7 & $861 \pm 43$ \\
\hline
\end{tabular}

6. Durée de la gestation (fig. I)

Elle a été établie d'après deux séries de données, celle de notre élevage et celle d'un élevage du Pays basque : la femelle est mise au mâle pendant 24 heures, ou bien des poursuites précédant l'accouplement sont observés.

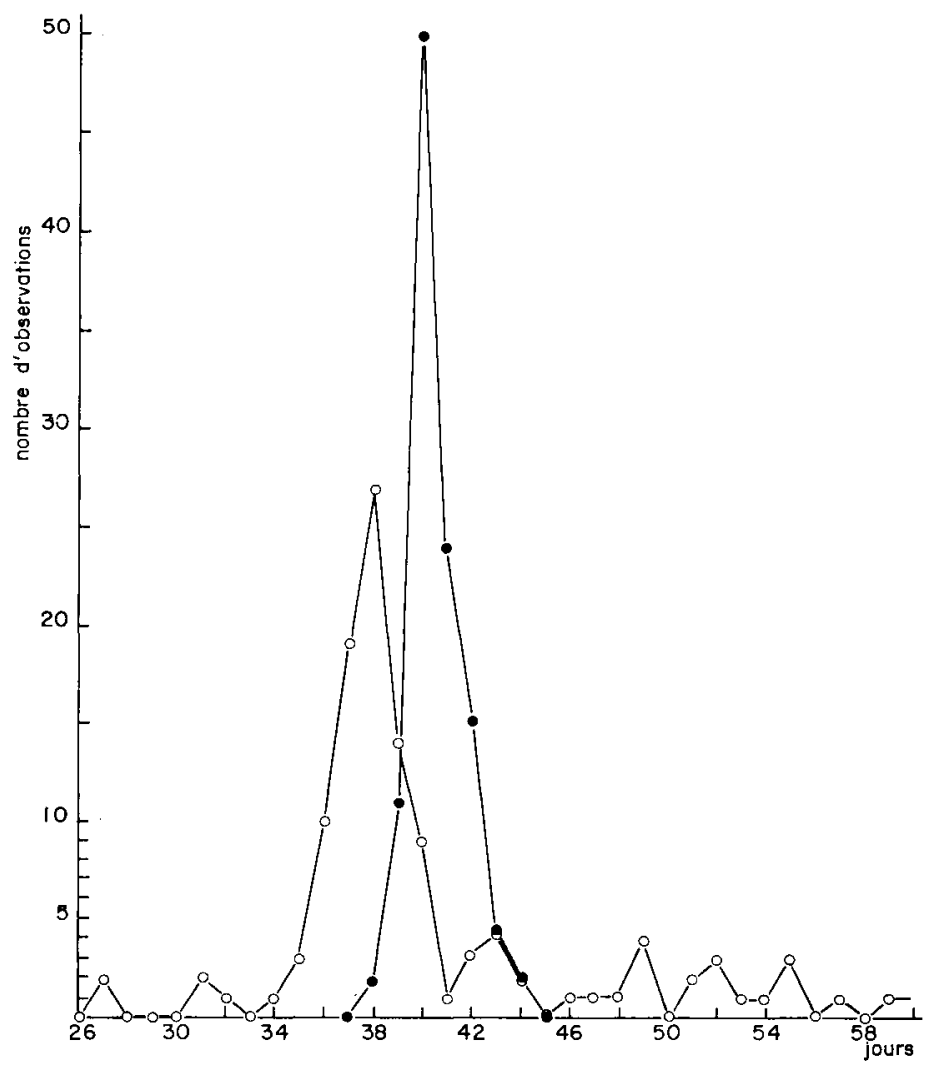

FIG. I. - Courbes de fréquence de l'intervalle entre les mises bas o-—o, et de la durée de la gestation 
A partìr de 43 observations faites dans notre élevage la durée de la gestation est de $4 \mathrm{I}, \mathrm{I} \pm 0,2$ jour; elle est de 40,2 \pm o, I jours dans l'élevage du Pays basque (66 observations).

La différence entre ces deux séries de données est significative. Il est possible qu'elle soit due à 1'imprécision de 24 heures concernant le moment exact de l'accouplement ou à une différence raciale.

Un cas aberrant a été observé où une hase a mis bas 52 jours après l'accouplement, de 2 levrauts mort-nés.

\section{Existence d'une superfcetation naturelle}

Elle a été mise en évidence à partir de la courbe de fréquence des intervalles entre deux mises bas successives et d'une laparotomie exploratrice réalisée chez une femelle gestante.

a) Chez les femelles laissées en permanence avec un mâle, le polygone de fréquence des intervalles entre deux mises bas (fig. I) a un mode de $3^{8}$ jours, alors que la durée de la gestation est de 40,5 jours. Le nombre d'observations où les intervalles entre deux mises bas et la durée de la gestation sont inférieurs ou égaux à 38 jours, représente respectivement 66 p. 100 et 2 p. Ioo du nombre total $(\mathrm{P}<0,0$ I).

Donc, il semble que dans au moins 66 p. roo des cas, ait lieu un accouplement fécond, 2 à 13 jours, le plus souvent 2 à 3 jours, avant la mise bas.

b) Chez une femelle ayant mis bas de 3 levrauts, une laparotomie exploratrice réalisée le même jour, a montré à droite 2 cicatrices placentaires sur la corne utérine, 2 corps jaunes anciens et 2 corps jaunes néo-formés provenant d'ovulations récentes sur l'ovaire, à gauche, I cicatrice placentaire sur la corne utérine, I corps jaune et I ovulation récente.

La perfusion de l'oviducte gauche a donné une morula de i 6 cellules, qui pourrait correspondre à un âge de 2 à 3 jours, après la fécondation par analogie avec Lepus Californicus (BARNEs, Ig66) et avec le Lapin.

Trente-huit jours après la laparotomie, la hase a mis bas de 2 levrauts correspondant aux 2 ovulations observées sur l'ovaire droit.

\section{DISCUSSION}

- La durée de la saison sexuelle observée en captivité correspond tout à fait à celle existant dans la nature. En effet, chez les mâles, la spermatogenèse commence au début décembre (FLUX, I965), les femelles primipares ou multipares ont leur première portée annuelle pendant la seconde moitié de janvier (LLOYD, I968), ce qui situe les premiers accouplements entre le to et le 20 décembre.

- La variation de la taille de la portée au cours de l'année a été rapportée par plusieurs auteurs (REYNOLDS, I950; RACZYNSKI, I964); il est probable que la durée d'éclairement intervient dans ce phénomène ; en effet, une jeune hase primipare de 6 mois mise sous une photopériode claire de 16 heures par 24 heures a mis bas de 4 jeunes en janvier et de 3 jeunes en mars, ce qui représente des nombres jamais observés pour une première ou une seconde portée. 
- La durée de la gestation déterminée par $\mathrm{BLOCH}$ et al. (I954) comme étant de 42 jours d'après un très petit nombre de cas est de 40,5 jours, ceci à partir de Iog observations.

Les durées beaucoup plus courtes, 30 à 38 jours, rapportées dans la littérature (BUFFON, I749 ; DESMERET, I820... relevées par FRAGUGLIONE, I96I) sont sans doute explicables par la présence d'une superfœetation qui réduit l'intervalle de temps entre deux mises bas successives.

Les durées plus longues (60 jours pour LIENHART, I940) correspondent peut-être à des intervalles entre mises bas comprenant une pseudo-gestation de $\mathrm{I} 8$ jours environ (BARnes, I966, chez Lepus Californicus) et une gestation de 30 à 4I jours.

- La fréquence d'une superfoctation, observée dans 66 p. Ioo des cas dans l'élevage semble plus élevée que dans la nature; FLUX (I967) n'observe que I3 p. Ioo de femelles en fin de gestation avec des œufs fécondés dans l'oviducte.

Si BLocr et al. (I967) avaient démontré 1'existence simultanée d'un fœetus à terme et de deux œufs fécondés à quatre cellules, ils n'avaient pu montrer si cette fécondation permettait le développement de jeunes viables; de plus, il n'était pas possible d'après ce travail de savoir si les oufs fécondés se trouvaient du côté de la corne utérine pleine. Les trois observations faites par FLUX (Ig67) correspondaient à trois gestations unilatérales avec présence d'œufs fécondés du côté de la corne vide. Dans le cas observé ici, il apparaît que la fécondation des œufs est possible alors que les deux cornes contiennent des fotus à terme.

Reçu pour publication en décembre 1969.

\section{REMERCIEMENTS}

Ce travail a été entrepris sur la demande et avec l'aide du Conseil supérieur de la Chasse à qui nous exprimons nos remerciements.

Nous tenons à remercier tout particulièrement M. Mondiet, à Jaxtou, Basses-Pyrénées, qui nous a aimablement fourni des renseignements sur son élevage.

\section{SUMMARY}

\section{OBSERVATIONS ON REPRODUC'TION IN THE CAP'TIVE BROWN HARE} (LEPUS EUROPAEUS PAILAS)

The following observations derive from a three-year cage breeding of brown hares.

The length of the breeding season is January to August or September, as it is for wild hares. Litter size reaches a maximum between May and July.

Gestation length is $40-4 \mathrm{I}$ days. Natural superfotation resulting from fertile matings during pregnancy was recorded in 60 per Ioo parturitions, which seems a much higher frequency rate than that of wild animals. 


\section{RÉFÉRENCES BIBIIOGRAPHIQUES}

BARnes R. D., I966. Natural reproduction, superovulation and insemination of black-tailed Jackrabbits. Ph. D. Thesis Univ. California, $53 \mathrm{pp.}$

Bloch S., Hediger H., Muller L., 1954. Probleme der Fortpflanzung der lieldhasen. Rev. Suisse Biol., 61, 485-490.

Bloch S., Hediger H., Lloyd H. G., Mijller C., Strauss F., ig67. Beobachtungen zur superfetation beim Feldhasen (Lepus europaeus). Zeits. f. Jagdwissensch., 13, 49-5 I.

Futx E. C., I965. Timing of the breeding season in the hare, Lepus europaeus and rabbit, Oryctolagus cuniculus. Mammalia, 29, 557-562.

Fuvx E. C., 1967. Reproduction and body weights of the hare, Lepus europaeus pallas in New Zealand. N. Z.J. Sci., 10, 357-401.

Fragilione ID., I96I. Durata della gestazione nelle Lepre comnune. Diana, 10, I-2.

Kolosov A. M., I94I. Biologie sexuelle du Lièvre européen (Lepus europaeus) (en russe). Zool. Zh., 20, I 54-I 72.

Lienhart R., I940. Durée de la gestation chez le Lièvre. C. R. Soc. Biol., 133, 133-I35.

Lloyd H. G., 1968. Observations on breeding in the brown hare (Lepus europaeus) during the first pregnancy of the season. J. Zool. London, 156, $52 \mathrm{I}-528$.

Raczinski J., I964. Studies on the european Hare, V. Reproduction. Acta Theriol., 9, 305-352.

Reynolds J. K., I950. Observations on the reproduction of Lepus europaeus Pallas in Ontario. M. S. Thesis West Ontario Univ., $55 \mathrm{pp}$.

STIEve H. Von., 1952. Zur lortpflanzungsbiologie der europäischen Feldhasen (Lepus europaeus PaLLAS). Zool. Anz., 148, IOI-II4. 